\title{
Geodesic Mappings and Einstein Spaces
}

\author{
Irena Hinterleitner and Josef Mikeš
}

\begin{abstract}
In this paper we study fundamental properties of geodesic mappings with respect to the smoothness class of metrics. We show that geodesic mappings preserve the smoothness class of metrics. We study geodesic mappings of Einstein spaces.
\end{abstract}

Mathematics Subject Classification (2010). 53C21; 53C25; 53B21; 53B30.

Keywords. Geodesic mapping, smoothness class, Einstein space.

\section{Introduction}

First we study the general dependence of geodesic mappings of (pseudo-) Riemannian manifolds in dependence on the smoothness class of the metric. We present well known facts, which were proved by Beltrami, Levi-Civita, Weyl, Sinyukov, etc., see [1, 6, 9, 11, 12. In these results no details about the smoothness class of the metric were stressed. They were formulated "for sufficiently smooth" geometric objects.

In the last section we present proofs of some facts about geodesic mappings of Einstein spaces.

\section{Geodesic mapping theory for $V_{n} \rightarrow \bar{V}_{n}$ of class $C^{1}$}

Assume the (pseudo-) Riemannian manifolds $V_{n}=(M, g)$ and $\bar{V}_{n}=(\bar{M}, \bar{g})$ with metrics $g$ and $\bar{g}$, and Levi-Civita connections $\nabla$ and $\bar{\nabla}$, respectively. Here $V_{n}, \bar{V}_{n} \in C^{1}$, i.e. $g, \bar{g} \in C^{1}$ which means that their components $g_{i j}$, $\bar{g}_{i j} \in C^{1}$.

Definition 1. A diffeomorphism $f: V_{n} \rightarrow \bar{V}_{n}$ is called a geodesic mapping of $V_{n}$ onto $\bar{V}_{n}$ if $f$ maps any geodesic in $V_{n}$ onto a geodesic in $\bar{V}_{n}$.

The paper was supported by grant P201/11/0356 of The Czech Science Foundation, MSM 6198959214, MSM 0021630511 of the Council of the Czech Government, and by the project FAST-S-11-47 of the Brno University of Technology. 
Let there exist a geodesic mapping $f: V_{n} \rightarrow \bar{V}_{n}$. Since $f$ is a diffeomorphism, we can suppose local coordinate maps on $M$ or $\bar{M}$, respectively, such that locally, $f: V_{n} \rightarrow \bar{V}_{n}$ maps points onto points with the same coordinates, and $\bar{M}=M$. A manifold $V_{n}$ admits a geodesic mapping onto $\bar{V}_{n}$ if and only if the Levi-Civita equations

$$
\bar{\nabla}_{X} Y=\nabla_{X} Y+\psi(X) Y+\psi(Y) X
$$

hold for any tangent fields $X, Y$ and where $\psi$ is a differential form. If $\psi \equiv 0$ than $f$ is affine or trivially geodesic.

In local form: $\bar{\Gamma}_{i j}^{h}=\Gamma_{i j}^{h}+\psi_{i} \delta_{j}^{h}+\psi_{j} \delta_{i}^{h}$, where $\Gamma_{i j}^{h}\left(\bar{\Gamma}_{i j}^{h}\right)$ are the Christoffel symbols of $V_{n}$ and $\bar{V}_{n}, \psi_{i}$ are components of $\psi$ and $\delta_{i}^{h}$ is the Kronecker delta. Equations (1) are equivalent to the following equations

$$
\bar{g}_{i j, k}=2 \psi_{k} \bar{g}_{i j}+\psi_{i} \bar{g}_{j k}+\psi \bar{g}_{i k}
$$

where "," denotes the covariant derivative on $V_{n}$. It is known that

$$
\psi_{i}=\partial_{i} \Psi, \quad \Psi=\frac{1}{2(n+1)} \ln \left|\frac{\operatorname{det} \bar{g}}{\operatorname{det} g}\right|, \quad \partial_{i}=\partial / \partial x^{i} .
$$

Sinyukov [12] proved that the Levi-Civita equations are equivalent to

$$
a_{i j, k}=\lambda_{i} g_{j k}+\lambda_{j} g_{i k},
$$

where

$$
\text { (a) } a_{i j}=\mathrm{e}^{2 \Psi} \bar{g}^{\alpha \beta} g_{\alpha i} g_{\beta j} ; \quad \text { (b) } \lambda_{i}=-\mathrm{e}^{2 \Psi} \bar{g}^{\alpha \beta} g_{\beta i} \psi_{\alpha} \text {. }
$$

From (3) follows $\lambda_{i}=\partial_{i} \lambda=\partial_{i}\left(\frac{1}{2} a_{\alpha \beta} g^{\alpha \beta}\right)$. On the other hand [11, p. 63]:

$$
\bar{g}_{i j}=\mathrm{e}^{2 \Psi} \tilde{g}_{i j}, \quad \Psi=\frac{1}{2} \ln \left|\frac{\operatorname{det} \tilde{g}}{\operatorname{det} g}\right|, \quad\left\|\tilde{g}_{i j}\right\|=\left\|g^{i \alpha} g^{j \beta} a_{\alpha \beta}\right\|^{-1} .
$$

The above formulas are the criterion for geodesic mappings $V_{n} \rightarrow \bar{V}_{n}$ globally as well as locally.

\section{Geodesic mapping theory for $V_{n} \rightarrow \bar{V}_{n}$ of class $C^{2}$}

Let $V_{n}$ and $\bar{V}_{n} \in C^{2}$, then for geodesic mappings $V_{n} \rightarrow \bar{V}_{n}$ the Riemann and the Ricci tensors transform in this way

(a) $\quad \bar{R}_{i j k}^{h}=R_{i j k}^{h}+\delta_{k}^{h} \psi_{i j}-\delta_{j}^{h} \psi_{i k} ; \quad$ (b) $\quad \bar{R}_{i j}=R_{i j}-(n-1) \psi_{i j}$,

where $\psi_{i j}=\psi_{i, j}-\psi_{i} \psi_{j}$, and the Weyl tensor of projective curvature, which is defined in the following form $W_{i j k}^{h}=R_{i j k}^{h}+\frac{1}{n-1}\left(\delta_{k}^{h} R_{i j}-\delta_{j}^{h} R_{i k}\right)$, is invariant.

The integrability conditions of the Sinyukov equations (3) have the following form

$$
a_{i \alpha} R_{j k l}^{\alpha}+a_{j \alpha} R_{i k l}^{\alpha}=g_{i k} \lambda_{j, l}+g_{j k} \lambda_{i, l}-g_{i l} \lambda_{j, k}-g_{j l} \lambda_{i, k} .
$$

After contraction with $g^{j k}$ we get 12

$$
n \lambda_{i, l}=\mu g_{i l}-a_{i \alpha} R_{l}^{\alpha}+a_{\alpha \beta} R^{\alpha}{ }_{i l}{ }^{\beta}
$$

where $R^{\alpha}{ }_{i l}{ }^{\beta}=g^{\beta k} R^{\alpha}{ }_{i l k} ; R_{l}^{\alpha}=g^{\alpha j} R_{j l}$ and $\mu=\lambda_{\alpha, \beta} g^{\alpha \beta}$. 
4. Geodesic mapping between $V_{n} \in C^{r}(r>2)$ and $\bar{V}_{n} \in C^{2}$

Theorem 2. If $V_{n} \in C^{r}(r>2)$ admits geodesic mappings onto $\bar{V}_{n} \in C^{2}$, then $\bar{V}_{n} \in C^{r}$.

The proof of this Theorem follows from the following lemmas.

Lemma 3. Let $\lambda^{h} \in C^{1}$ be a vector field and $\varrho$ a function. If $\partial_{i} \lambda^{h}-\varrho \delta_{i}^{h} \in C^{1}$ then $\lambda^{h} \in C^{2}$ and $\varrho \in C^{1}$.

Proof. The condition $\partial_{i} \lambda^{h}-\varrho \delta_{i}^{h} \in C^{1}$ can be written in the following form

$$
\partial_{i} \lambda^{h}-\varrho \delta_{i}^{h}=f_{i}^{h}(x),
$$

where $f_{i}^{h}(x)$ are functions of class $C^{1}$. Evidently, $\varrho \in C^{0}$. For fixed but arbitrary indices $h \neq i$ we integrate (9) with respect to $d x^{i}$ :

$$
\lambda^{h}=\Lambda^{h}+\int_{x_{o}^{i}}^{x^{i}} f_{i}^{h}\left(x^{1}, \ldots, x^{i-1}, t, x^{i+1}, \ldots, x^{n}\right) d t,
$$

where $\Lambda^{h}$ is a function, which does not depend on $x^{i}$.

Because of the existence of the partial derivatives of the functions $\lambda^{h}$ and the above integrals (see [5, p. 300]), also the derivatives $\partial_{h} \Lambda^{h}$ exist; in this proof we don't use Einstein's summation convention. Then we can write (9) for $h=i$ :

$$
\varrho=-f_{h}^{h}+\partial_{h} \Lambda^{h}+\int_{x_{o}^{i}}^{x^{i}} \partial_{h} f_{i}^{h}\left(x^{1}, \ldots, x^{i-1}, t, x^{i+1}, \ldots, x^{n}\right) d t .
$$

Because the derivative with respect to $x^{i}$ of the right-hand side of (10) exists, the derivative of the function $\varrho$ exists, too. Obviously $\partial_{i} \varrho=\partial_{h} f_{i}^{h}-\partial_{i} f_{h}^{h}$, therefore $\varrho \in C^{1}$ and from (9) follows $\lambda^{h} \in C^{2}$.

In a similar way we can prove the following: if $\lambda^{h} \in C^{r}(r \geq 1)$ and $\partial_{i} \lambda^{h}-\varrho \delta_{i}^{h} \in C^{r}$ then $\lambda^{h} \in C^{r+1}$ and $\varrho \in C^{r}$.

Lemma 4. If $V_{n} \in C^{3}$ admits a geodesic mapping onto $\bar{V}_{n} \in C^{2}$, then $\bar{V}_{n} \in C^{3}$.

Proof. In this case Sinyukov's equations (3) and (8) hold. According to the assumptions $g_{i j} \in C^{3}$ and $\bar{g}_{i j} \in C^{2}$. By a simple check-up we find $\Psi \in C^{2}$, $\psi_{i} \in C^{1}, a_{i j} \in C^{2}, \lambda_{i} \in C^{1}$ and $R_{i j k}^{h}, R^{h}{ }_{i j}{ }^{k}, R_{i j}, R_{i}^{h} \in C^{1}$.

From the above-mentioned conditions we easily convince ourselves that we can write equation (8) in the form (9), where $\lambda^{h}=g^{h \alpha} \lambda_{\alpha} \in C^{1}, \varrho=\mu / n$ and $f_{i}^{h}=\left(-\lambda^{\alpha} \Gamma_{\alpha i}^{h}-g^{h \gamma} a_{\alpha \gamma} R_{i}^{\alpha}+g^{h \gamma} a_{\alpha \beta} R^{\alpha}{ }_{i \gamma}{ }^{\beta}\right) / n \in C^{1}$.

From Lemma 3 follows that $\lambda^{h} \in C^{2}, \varrho \in C^{1}$, and evidently $\lambda_{i} \in C^{2}$. Differentiating (3) twice we convince ourselves that $a_{i j} \in C^{3}$. From this and formula (5) follows that also $\Psi \in C^{3}$ and $\bar{g}_{i j} \in C^{3}$.

Further we notice that for geodesic mappings between $V_{n}$ and $\bar{V}_{n}$ of class $C^{3}$ holds the third set of Sinyukov equations:

$$
(n-1) \mu_{, k}=2(n+1) \lambda_{\alpha} R_{k}^{\alpha}+a_{\alpha \beta}\left(2 R_{k,}^{\alpha}{ }^{\beta}-R_{, k}^{\alpha \beta}\right) .
$$


If $V_{n} \in C^{r}$ and $\bar{V}_{n} \in C^{2}$, then by Lemma 4, $\bar{V}_{n} \in C^{3}$ and (11) hold. Because Sinyukov's system (3), (8) and (11) is closed, we can differentiate equations (3) $(r-1)$ times. So we convince ourselves that $a_{i j} \in C^{r}$, and also $\bar{g}_{i j} \in C^{r}\left(\equiv \bar{V}_{n} \in C^{r}\right)$.

Remark 5. Because for holomorphically projective mappings of Kähler (and also hyperbolic and parabolic Kähler) spaces hold equations analogical to (3) and (8), see [7, 9, 12, from Lemma 3 follows an analog to Theorem 2 for these mappings.

\section{On geodesic mappings of Einstein spaces}

Geodesic mappings of Einstein spaces were studied by many authors starting with A.Z. Petrov (see [10]). Einstein spaces $V_{n}$ are characterized by the condition $R i c=$ const $\cdot g$, so $V_{n} \in C^{2}$ would be sufficient. But many properties of Einstein spaces appear when $V_{n} \in C^{3}$ and $n>3$. An Einstein space $V_{3}$ is a space of constant curvature.

We continue with geodesic mappings of Einstein spaces $V_{n} \in C^{3}$. On basis of Theorem 2 it is natural to suppose that $\bar{V}_{n} \in C^{3}$. In 1978 (see PhD thesis [3] and 4]) Mikeš proved that under these conditions the following theorem holds:

Theorem 6. If the Einstein space $V_{n}$ admits a nontrivial geodesic mapping onto a (pseudo-) Riemannian space $\bar{V}_{n}$, then $\bar{V}_{n}$ is an Einstein space.

Proof. Let the Einstein space $V_{n} \in C^{3}$ (for which $R_{i j}=-K(n-1) g_{i j}$ ) admit a nontrivial geodesic mapping onto $\bar{V}_{n} \in C^{2}$. Then the Sinyukov equations (3) hold; their integrability conditions have the form (77). Taking (3) into account, we differentiate (7) with respect to $x^{m}$, contract the result with $g^{l m}$, and then we alternate with respect to $i, k$. By (9), we get $\lambda_{\alpha} R_{i j k}^{\alpha}=g_{i j} \xi_{k}-g_{i k} \xi_{j}$, where $\xi_{i}$ is some vector. Contracting the latter with $g^{i j}$ and using (9) we see that $\xi_{i}=K \lambda_{i}$, that is, the formula reads $\lambda_{\alpha} R_{i j k}^{\alpha}=K\left(g_{i j} \lambda_{k}-g_{i k} \lambda_{j}\right)$.

We contract (7) with $\lambda^{l}$. Considering the last formula, we get

$$
g_{k i} \Lambda_{j \alpha} \lambda^{\alpha}+g_{k j} \Lambda_{i \alpha} \lambda^{\alpha}-\lambda_{i} \Lambda_{j k}-\lambda_{j} \Lambda_{i k}=0,
$$

where $\Lambda_{i j}=\lambda_{i, j}-K a_{i j}$. It is easy to show that $\lambda^{\alpha} \Lambda_{\alpha i}=\mu \lambda_{i}$, where $\mu$ is a function. Since $\lambda_{i} \neq 0$, we find from (12) that

$$
\lambda_{i, j}=\mu g_{i j}+K a_{i j} .
$$

Differentiating (4b) and considering (2), (3), (4), it is easy to get the following equation:

$$
\psi_{i j} \equiv \psi_{i, j}-\psi_{i} \psi_{j}=\bar{K} g_{i j}-K \bar{g}_{i j},
$$

where $\bar{K}$ is a function. Then from (6b), by virtue of the last relation, and considering $R_{i j}=-K(n-1) g_{i j}$, we get that $\bar{R}_{i j}=(n-1) \bar{K} \bar{g}_{i j}$. Hence $\bar{V}_{n}$ is an Einstein space. The theorem is proved. 
Theorem [6] was proved "locally" but it is easy to show that when the domain of validity of equations (14) borders with a domain where $\psi_{i} \equiv 0$, then in this domain $\psi_{i} \equiv 0$. Assume a point $x_{0}$ on the borders between these domains, then $\psi_{i}\left(x_{0}\right)=0$ and $\psi_{i j}=0$. Indeed a) If $K \neq 0$ or $\bar{K} \neq 0$ then $\bar{g}_{i j}\left(x_{0}\right)=\bar{K} / K g_{i j}\left(x_{0}\right)$. From these properties follows that the system of equations (2) and (14) has a unique solution $\bar{g}_{i j}=\bar{K} / K g_{i j}$ and $\psi_{i}=0$. b) If $K=\bar{K}=0$ then equations (14): $\psi_{i, j}=\psi_{i} \psi_{j}$ have a unique solution for $\psi_{i}\left(x_{0}\right)=0: \psi_{i}=0$.

This Theorem was used for geodesic mappings of 4-dimensional Einstein spaces (Mikeš, Kiosak [8]) and to find metrics of Einstein spaces that admit geodesic mappings (Formella, Mikeš [2]), etc.

\section{References}

[1] L. P. Eisenhart, Non-Riemannian Geometry. Princeton Univ. Press. 1926. Amer. Math. Soc. Colloquium Publications 8 (2000).

[2] S. Formella, J. Mikeš, Geodesic mappings of Einstein spaces. Szczecińske rocz. naukove, Ann. Sci. Stetinenses. 9 I. (1994) 31-40.

[3] J. Mikeš, Geodesic and holomorphically projective mappings of special Riemannian spaces. PhD thesis, Odessa, 1979.

[4] J. Mikeš, Geodesic mappings of Einstein spaces. Math. Notes 28 (1981) 922-923; transl. from Mat. Zametki 28 (1980) 935-938.

[5] L. D. Kudrjavcev, Kurs matematicheskogo analiza. Moscow, Vyssh. skola, 1981.

[6] J. Mikeš, Geodesic mappings of affine-connected and Riemannian spaces. J. Math. Sci., New York 78 (1996) 311-333.

[7] J. Mikeš, Holomorphically projective mappings and their generalizations. J. Math. Sci., New York 89 (1998) 1334-1353.

[8] J. Mikeš, V.A. Kiosak, On geodesic maps of four dimensional Einstein spaces. Odessk. Univ. Moscow: Archives at VINITI, 9.4.82, No. 1678-82, (1982).

[9] J. Mikeš, A. Vanžurová, I. Hinterleitner, Geodesic mappings and some generalizations. Palacky University Press, 2009.

[10] A. Z. Petrov, New methods in the general theory of relativity. M., Nauka, 1966.

[11] Zh. Radulovich, J. Mikeš, M. L. Gavril'chenko, Geodesic mappings and deformations of Riemannian spaces. (Russian) Podgorica: CID. Odessa: OGU, 1997.

[12] N. S. Sinyukov, Geodesic mappings of Riemannian spaces. M., Nauka, 1979.

Irena Hinterleitner

Dept. of Math., Brno University of Technology,

Žižkova 17, CZ 60200 Brno, Czech Republic

e-mail: hinterleitner.i@fce.vutbr.cz

Josef Mikeš

Dept. of Algebra and Geometry, Palacky University, 17. listopadu 12, CZ 77146 Olomouc, Czech Republic

e-mail: josef.mikes@upol.cz 